\title{
Emphysematous cholecystitis
}

\author{
Miguel F. Carrascosa MD, José R. Salcines-Caviedes MD
}
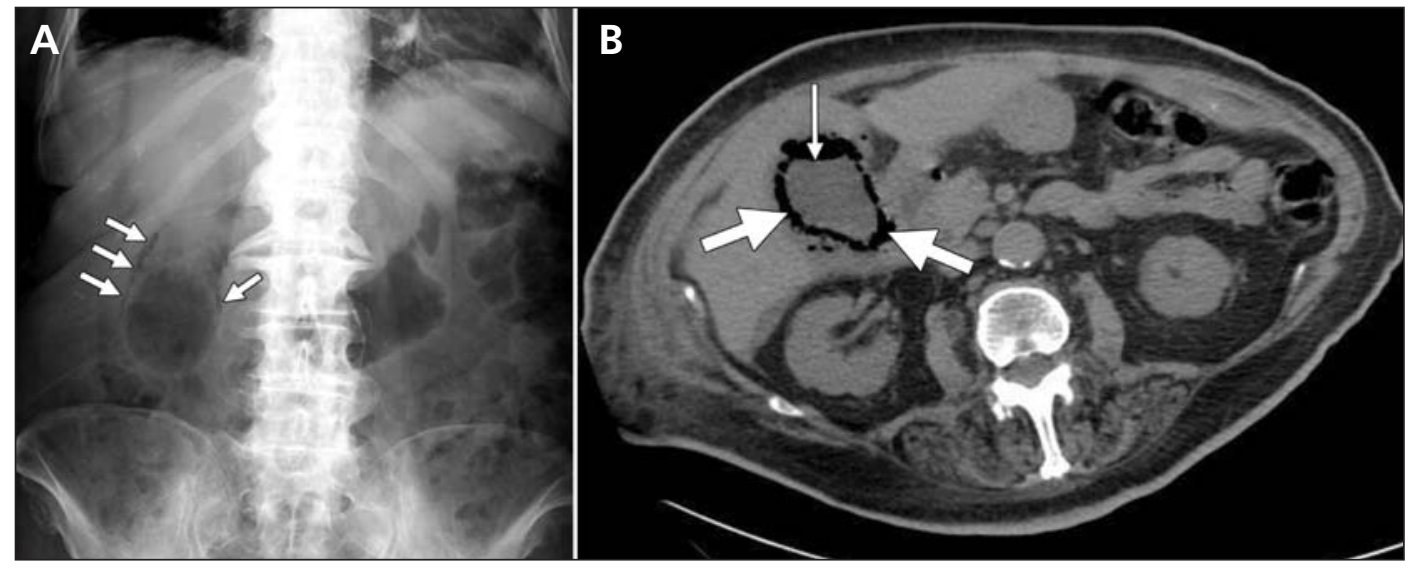

Competing interests: None declared.

This article has been peer reviewed.

Affiliations: From the Internal Medicine Department (Carrascosa) and Digestive Diseases

Section (SalcinesCaviedes), Hospital of Laredo, Laredo, Spain

Correspondence to: Dr. Miguel F. Carrascosa, miguel.carrascosa@scsalud.es

Figure 1: (A) Plain-film radiograph showing air in the lumen and wall of the enlarged gallbladder of an 87 CMAJ 2012. DOI:10.1503 year-old woman with abdominal pain and fever (arrows). (B) Computed tomography of the abdomen, showing an air-liquid level in the lumen (thin arrow) and gas in the wall (thick arrows) of the gallbladder.

$\Lambda$ n 87-year-old woman presented with fever and drowsiness of two days' duration. She had a history of diabetes mellitus, atrial fibrillation and post-stroke global aphasia. On examination, she had diffuse abdominal tenderness. Because laboratory data showed leukocytosis and bacteriuria, she was thought to have a urinary tract infection. Blood and urine cultures were obtained, and she was given intravenous amoxicillin clavulanate. Her fever resolved, but hypochondrial guarding developed on her right side on the third day after admission. A plain radiograph of the abdomen with the patient in a supine position suggested the presence of a dilated gallbladder with air in the lumen and wall (Figure 1A). Computed tomography showed an air-liquid level in the gallbladder lumen, gas dissecting the entire gallbladder wall, and pneumoperitoneum (Figure 1B). Cholecystectomy was not performed because the patient was thought to be too frail. Instead, she was given piperacillin tazobactam intravenously, and percutaneous drainage of the gallbladder was subsequently performed. Although the urine culture grew Escherichia coli, blood and bile cultures from the drainage were negative. The patient recovered and was able to eat at discharge.

Emphysematous cholecystitis is a life-threatening form of acute cholecystitis that is more com- mon in older patients with diabetes mellitus than in the general population. ${ }^{1}$ The term refers to the presence of gas in the gallbladder lumen or wall or in the pericholecystic fluid. Clostridium spp, $E$. coli, Klebsiella spp and anaerobic streptococci are the most common gas-forming organisms that cause this disorder., Emphysematous cholecystitis is a consequence of a primary ischemic injury, and the main predisposing conditions are atherosclerosis, arterial embolism, vasculitis and systemic hypoperfusion. ${ }^{1,3}$ Although emergency cholecystectomy is the treatment of choice, percutaneous cholecystostomy can be effective if surgery is contraindicated or the patient is at high risk of complications or death. ${ }^{1,4}$ The mortality associated with emphysematous cholecystitis is $15 \%$, compared with $4 \%$ for acute, non-emphysematous cholecystitis. ${ }^{1}$ Prompt clinical suspicion and appropriate abdominal imaging are crucial in making the correct diagnosis.

\section{References}

1. Moanna A, Bajaj R, del Rio C. Emphysematous cholecystitis due to Salmonella derby. Lancet Infect Dis 2006;6:118-20.

2. Chiu HH, Chen CM, Mo LR. Emphysematous cholecystitis. Am J Surg 2004;188:325-6.

3. Bouras G, Lunca S, Vix M, et al. A case of emphysematous cholecystitis managed by laparoscopic surgery. JSLS 2005;9:478-80.

4. Wu JM, Lee CY, Wu YM. Emphysematous cholecystitis. Am J Surg 2010;200:e53-4. 